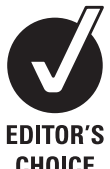

CHOICE

\title{
Non-cystic fibrosis bronchiectasis: diagnosis and management in 21 st century
}

\author{
Pieter Goeminne, Lieven Dupont
}

Laboratory of Pneumology, University Hospital Gasthuisberg, Katholieke Universiteit Leuven, Belgium

\section{Correspondence to}

Professor L Dupont, University Hospital Gasthuisberg,

Respiratory Medicine, Herestraat 49, B-3000 Leuven, Belgium;

lieven.dupont@uz.kuleuven.be

Received 11 September 2009 Accepted 31 May 2010

\begin{abstract}
Bronchiectasis is permanently dilated airways caused by chronic bronchial inflammation secondary to inappropriate clearance of various micro-organisms and recurrent infections in the airways. At diagnosis, one should search for the underlying disease process, most of the time excluding cystic fibrosis (CF). However, in a substantial number of patients no cause is found. Next, patients need individualised therapy and follow-up by monitoring of their symptoms. Useful tools are the Leicester Cough Questionnaire and the Sputum Colour Chart. Screening patients for bacterial colonisation on a regular basis seems to be equally important, as many patients become colonised by pathogenic microorganisms. Treatment for non-cystic fibrosis bronchiectasis differs in certain aspects from cystic fibrosis bronchiectasis and often lacks evidence. Overall, bronchiectasis is an underestimated disease, not only in prevalence and incidence, but also in its ability to cause morbidity and mortality. Further research into the underlying pathophysiological mechanisms and trials evaluating new treatments are an absolute necessity.
\end{abstract}

\section{INTRODUCTION}

Bronchiectasis is defined as permanently dilated airways due to chronic bronchial inflammation caused by inappropriate clearance of various microorganisms and recurrent or chronic infection, ${ }^{1}$ and it was first described by Rene Theophile Laënnec in $1819^{2}$ (box 1). Bronchiectasis is characterised by thickening of the bronchial wall, leading to increased sputum production and chronic cough. Overall, bronchiectasis most commonly occurs as a sequel to infection, certainly in less developed countries, but, in Europe, it is often seen in patients with cystic fibrosis (CF). ${ }^{1}$ In this review, we will focus on the diagnosis of bronchiectasis in patients in whom CF has been excluded (non-cystic fibrosis bronchiectasis (NCFB)) and discuss a number of controversies in the current management of this disorder.

\section{PREVALENCE AND INCIDENCE}

Bronchiectasis is diagnosed with increasing frequency, which is due to increased use of highresolution chest CT (HRCT). Post infectious causes used to be the overall leading source of bronchiectasis in the world, but this is now less common in areas where tuberculosis and childhood pneumonia are less prevalent. Vaccination programmes have further reduced the contribution of pertussis and measles to bronchiectasis in these areas. In less developed countries, post infectious bronchiectasis is still the leading cause because of poor access to healthcare and a high infection rate. Diagnosing post infectious causes may be difficult because of inaccurate recall and reporting and the ability of most people to make a full recovery without lasting pulmonary symptoms after these infections. ${ }^{4}$ A good example of poor access to healthcare is the Alaskan Eskimo population living in the Yukon-Kuskokwim Delta. Although they only account for $20 \%$ of the total Alaska Native population, they have had a disproportionately high prevalence of NCFB during the last few decades, ranging from 10 to 20 per 1000 . This high prevalence is probably the result of high rates of severe respiratory infections at an early age, rather than a racial or geographical predisposition. Reasons for this high rate of respiratory infection are isolation, poverty, household overcrowding and poor living conditions, which correlate with ethnicity and geographic location. ${ }^{5}$ Few data are available on overall prevalence. In Finland an incidence of 3.9 per 100000 per year was reported in the overall population (0.49 per 100000 per year in the under 15 year group), whereas in New Zealand the prevalence was estimated to be 3.7 per 100000 per year under the age of 15 years. In the USA, a prevalence of 52 per 100000 has been reported. ${ }^{6}$

\section{SIGNS AND SYMPTOMS}

The symptoms and clinical course of NCFB are variable. Some patients have no symptoms at all or only during exacerbations, whereas others have them daily. Patients complain of chronic cough, sputum production and lethargy, but haemoptysis, chest pain, weight loss, bronchospasms, dyspnoea and decreased exercise tolerance may be present too. A number of patients have frequent exacerbations of their symptoms due to NCFB. Exacerbation rates in some Western countries (Ireland, UK and North America) were found to be 1.5 per year. Exacerbations can be defined as patients reporting four or more of the following symptoms: change in sputum production, increased dyspnoea, increased cough, fever over $38^{\circ} \mathrm{C}$, increased wheezing, decreased exercise tolerance, fatigue, malaise, lethargy, reduced pulmonary function, changes in chest sounds or radiographic changes consistent with a new infectious process. ${ }^{7}$ Loss of lung function has been demonstrated in patients with non-smoking NCFB, with an average decline of $\sim 50 \mathrm{ml} /$ year in forced expiratory volume in $1 \mathrm{~s}\left(\mathrm{FEV}_{1}\right)$. Factors associated with disease progression are a history of severe exacerbations, chronic colonisation with Pseudomonas aeruginosa, and evidence of systemic inflammation. A FEV 1 decline of $123 \mathrm{ml} /$ year was reported in patients with chronic Ps aeruginosa colonisation..$^{8} 9$ Severe NCFB may also induce pulmonary hypertension and right and left ventricular systolic and diastolic dysfunction. ${ }^{10}$ 


\section{Box 1 Brief history of bronchiectasis}

Laënnec first described bronchiectasis in 1819. He stated that '...the dilated bronchi lose their natural shape, and present themselves under the form of a cavity, capable of containing a hemp-seed, a cherry-stone, an almond, or even a walnut'. A famous patient with non-cystic fibrosis bronchiectasis was William Osler, who died in 1919 as a result of lung abscess and empyema, which were complications of the pneumonia he contracted after a motor drive from Newcastle to Oxford. For many years, he had severe chest infections suggesting underlying bronchiectasis. This was later confirmed by Dr George Gibson, who performed the post-mortem examination. ${ }^{3}$ Other landmarks in the history of bronchiectasis were the introduction of contrast bronchography by Jean Athanase Sicard, permitting the precise imaging of the destructive changes in airways. In the 1950s, Lynne Reid linked bronchography with pathological specimens. ${ }^{1}$

To allow clinicians to measure directly the impact of the disease on daily life, health-related quality of life questionnaires have been developed for chronic respiratory diseases, such as the St George's Respiratory Questionnaire (SGRQ). Data show that the SGRO is a valid outcome measure for bronchiectasis that distinguishes between different levels of impaired health and appears to be sensitive to spontaneous changes in health over a 6-month period. ${ }^{11}$ The Leicester Cough Questionnaire (LCQ) is a tool for assessing cough severity, the dominant symptom of bronchiectasis, in a standardised way. It is a 19-item questionnaire validated for use in the assessment of NCFB. It is able to discriminate disease severity and to document treatment response. $^{12}$

\section{PATHOPHYSIOLOGY}

The aetiology of NCFB is variable, but the common pathophysiological pathway represents a vicious cycle containing three important elements: inflammation, infection and damage (by enzymatic components). Local airway colonisation with micro-organisms that cannot be properly cleared by the host because of various underlying diseases leads to infection that causes an inflammatory reaction. Inflammation protects against invasion of foreign material, but when this response fails to eliminate the aggressor, the inflammation may turn chronic, inducing bronchial wall damage and irreversible dilatation. The airway inflammatory response triggered by this bacterial infection appears to be excessive in relation to the bacterial burden and may persist even after the infection has been controlled, resulting in an increased production of proinflammatory cytokines and/or uncontrolled activation of effector cells. ${ }^{13}$

\section{DIAGNOSIS OF NCFB}

\section{Causes and related diseases}

Different underlying conditions causing NCFB have been identified and are listed in table 1 . We will discuss some of them in more detail.

\section{Postinfectious}

Several respiratory infections can cause bronchiectasis, including measles, pertussis and tuberculosis, but also viruses (HIV, paramyxovirus, adenovirus and influenza), Gram-negative bacteria
Table 1 Category and causes of non-cystic fibrosis bronchiectasis

\begin{tabular}{|c|c|}
\hline Category & Causes \\
\hline \multirow[t]{4}{*}{ Postinfectious } & Viral \\
\hline & Bacterial \\
\hline & Fungal \\
\hline & Atypical mycobacteria $^{15}$ \\
\hline \multirow{2}{*}{\multicolumn{2}{|c|}{$\begin{array}{l}\text { ABPA } \\
\text { COPD }^{16-18}\end{array}$}} \\
\hline & \\
\hline \multicolumn{2}{|l|}{ Idiopathic } \\
\hline \multirow[t]{5}{*}{ Traction } & Post-tuberculosis fibrosis \\
\hline & Post-radiation fibrosis \\
\hline & Fibrosis (eg, sarcoidosis) \\
\hline & Collagen vascular diseases \\
\hline & $\begin{array}{l}\text { Twisting or displacement of the airways } \\
\text { after lobar resection }\end{array}$ \\
\hline \multirow[t]{2}{*}{ Inhalation/aspiration } & Inhalation of corrosive substances \\
\hline & Aspiration of foreign body \\
\hline \multirow[t]{3}{*}{ Obstruction } & Benign bronchogenic tumours \\
\hline & Broncholithiasis \\
\hline & Enlarged lymph nodes \\
\hline \multirow[t]{2}{*}{ Amyloidosis $^{22}$} & Nodular pulmonary amyloidosis \\
\hline & Secondary amyloidosis \\
\hline \multicolumn{2}{|l|}{ Coeliac disease $^{28}$} \\
\hline \multicolumn{2}{|l|}{ Endometriosis $^{30}$} \\
\hline \multicolumn{2}{|l|}{ Yellow nail syndrome } \\
\hline \multicolumn{2}{|l|}{ Young's syndrome ${ }^{3536}$} \\
\hline \multirow[t]{8}{*}{ Immunological abnormalities } & Primary \\
\hline & Common variable immune deficiency \\
\hline & Congenital agammaglobulinaemia \\
\hline & Hyper lgE (Job) syndrome $e^{14}$ Etc. \\
\hline & Secondary \\
\hline & Chemotherapy \\
\hline & Immunosuppressive therapy \\
\hline & $\begin{array}{l}\text { Cancer (eg, chronic lymphatic } \\
\text { leukaemia) }\end{array}$ \\
\hline \multirow[t]{10}{*}{ Congenital abnormalities } & Anatomic \\
\hline & Scoliosis, pectus excavatum ${ }^{19}$ \\
\hline & Marfan syndrome \\
\hline & $\begin{array}{l}\text { Tracheobronchomegaly } \\
\text { (Mounier-Kuhn syndrome) }\end{array}$ \\
\hline & Pulmonary sequestration \\
\hline & Williams-Campbell syndrome \\
\hline & Other \\
\hline & $\begin{array}{l}\text { Primary ciliary dyskinesia (Kartagener } \\
\text { syndrome) }\end{array}$ \\
\hline & $\alpha 1$-antitrypsin deficiency \\
\hline & Defective $\mathrm{ENaC}$ protein ${ }^{20}$ \\
\hline \multicolumn{2}{|l|}{ Diffuse panbronchiolitis ${ }^{21}$} \\
\hline \multicolumn{2}{|l|}{ Relapsing polychondritis } \\
\hline \multicolumn{2}{|l|}{ Rheumatoid arthritis ${ }^{23-26}$} \\
\hline \multicolumn{2}{|l|}{ Systemic lupus erythematosus ${ }^{27}$} \\
\hline \multicolumn{2}{|l|}{ Sjögren ${ }^{29}$} \\
\hline Ankylosing spondylitis & \\
\hline Inflammatory bowel disease ${ }^{31-34}$ & \\
\hline
\end{tabular}

$\mathrm{ABPA}$, allergic brochopulmonary aspergillosis; COPD, chronic obstructive pulmonary disease.

( $P$ s aeruginosa, Haemophilus influenzae) and other atypical mycobacteria. NCFB secondary to Mycobacterium avium complex infection is a typical feature of the Lady Windermere syndrome. Patients are mostly older, immunocompetent women with no smoking history or pulmonary disease. Bronchiectasis is found typically in the middle lobe and lingula, which are predisposed to non-specific inflammation because of their particular anatomical structures and absence of collateral ventilation. It is hypothesised that they voluntary suppress coughing and are 
insufficiently clearing secretions. This focus of inflammation then predisposes to $M$ avium complex. ${ }^{15}$

Other pulmonary non-tuberculous mycobacteria (NTM) may cause pulmonary disease such as NCFB, and it has been recognised that certain anthropometric features are more related to NTM disease. These include scoliosis, pectus excavatum, mitral valve prolapse, tall stature and lean body. ${ }^{19} \mathrm{~A}$ specific cause of bronchiectasis is allergic brochopulmonary aspergillosis. Aspergillus can proliferate in the proximal bronchi and induce an IgEmediated hypersensitivity, resulting in inflammatory reaction and mucus impaction. This can lead to bronchial wall damage and bronchiectasis. A central distribution is often seen due to impaction in the central airways.

\section{Congenital conditions}

Several congenital conditions other than CF have been associated with bronchiectasis. The most well-known is primary ciliary dyskinesia (also called Kartagener syndrome in the presence of situs inversus). Abnormal ciliary motion will lead to recurrent infections due to retention of secretions. A more recent discovery of a congenital cause is that of Fajac et al, ${ }^{20}$ who showed that some patients with idiopathic bronchiectasis carried a mutation in the $\mathrm{ENaC} \beta$ or $\gamma$ genes, giving a defective epithelial sodium channel $(\mathrm{ENaC})$ protein.

\section{Chronic obstructive pulmonary disease (COPD)}

More recently, a high incidence of bronchiectasis has been reported in COPD, suggesting a new aetiological group for NCFB. ${ }^{16}$ Bronchiectasis was found in $29-50 \%$ of the investigated patients. These patients had more dyspnoea and worse lung function than the bronchiectasis-free COPD group. ${ }^{17} 18$

\section{Inflammatory bowel disease}

Extraintestinal manifestations of ulcerative colitis are common, with chronic bronchitis and NCFB being the most common. Respiratory manifestations remain puzzling because they can develop in patients whose inflammatory bowel disease is under control by medical therapy or even after surgery. It may be that the common ancestry of the bowel and bronchial tree play an important role in this suggested link. ${ }^{31}{ }^{32}$ For Crohn's disease, pulmonary disease, bronchiectasis in particular, seems to be rare. $^{33} 34$

\section{Related diseases: reflux disease and Helicobacter pylori}

There is no direct evidence that $H$ pylori causes bronchiectasis, but there may be an indirect link through $H$ pylori products such as toxins, urease, catalase, phospholipidases, alcohol dehydrogenase, haemolysis, platelet activating factor and mucolytic factor. ${ }^{37-39}$ Tsang et al ${ }^{40}$ showed a high prevalence of $H$ pylori-specific IgG in patients with bronchiectasis compared with healthy subjects. In addition, the concentration of $H$ pylori-specific IgG correlated with disease activity. Up to $32 \%$ of bronchiectatic patients have upper gastrointestinal symptoms. On the other hand, no $H$ pylori was found in bronchoalveolar lavage (BAL) fluid in children with idiopathic NCFB. ${ }^{41}$

\section{Assessment of airway colonisation and infection}

Sputum culture is standard in evaluating airway colonisation and infection in NCFB. If sputum cannot be produced spontaneously, sputum induction could be used as an alternative. In patients unable to expectorate or those with negative sputum culture but unfavourable clinical outcome, a bronchoscopy is indicated and BAL or protected specimen brush can be performed. Microbiological analysis of sputum (spontaneous or induced) gives adequate results, equivalent to a protected specimen brush. BAL does not seem to provide any additional information compared with a protected specimen brush. Risk factors for colonisation with potential pathogenic microorganisms are varicose cystic bronchiectasis, $\mathrm{FEV}_{1}$ below $80 \%$, and a diagnosis before the age of 14 . In more than $60 \%$ of adult patients with stable bronchiectatic disease, the airways are colonised. The most prevalent bacteria are Hae influenzae, Pseudomonas spp. and Streptococcus pneumoniae. Similar rates were found in children. ${ }^{42-44}$ More importantly, resistance to certain antibiotic treatments can be serious, reinforcing the importance of periodical assessment of the airway flora. ${ }^{45}{ }^{46}$ Colonised patients have a more intense inflammatory reaction than noncolonised. $^{47}$

There is a significant role for NTM in bronchiectasis. Positive cultures are found in $2-10 \%$ of patients. ${ }^{148} 49$ Although it is more common in CF, patients with NCFB should be screened for NTM if there is an unexplained deterioration that is unresponsive to the usual treatment. Moreover, patients with bronchiectasis and NTM have a higher prevalence of coexisting Aspergillus-related lung disease. ${ }^{50}$ Chronic colonisation by other bacteria such as Burkholderia cepacia is quite rare in NCFB, but has been described..$^{51}$ To clinically evaluate bacterial colonisation, a recent study from Murray et $a l^{52}$ evaluated the usefulness of sputum colour as a clinical tool. They found that, in stable patients with bronchiectasis, sputum purulence correlated with bacterial colonisation. Mucoid sputa were rarely colonised, whereas purulent sputa were colonised in $86 \%$ of cases. Isolated bacteria were mainly Hae influenzae and Ps aeruginosa, followed by Strep pneumoniae, Staphylococcus aureus and Moraxella catarrhalis.

\section{Imaging}

The current classification is based on the work of Lynne Reid who classified bronchiectasis according to the degree of bronchial dilatation as well as the severity of bronchiolar obliteration. The first degree is cylindrical bronchiectasis, in which the bronchi are minimally dilated and they maintain a smooth and regular outline. More distal bronchi are plugged with thick and purulent material. The second group is named varicose bronchiectasis because of its resemblance to varicose veins. The bronchial outline is much more irregular and dilatation is greater than in the first degree. Finally, the most severe form of bronchiectasis is saccular or cystic deformation. It is characterised by progressive bronchial dilatation towards the periphery of the lung. ${ }^{53}$

In one study, bronchography-proven bronchiectasis showed that chest radiography was normal in only $7.1 \%$ of patients. Common findings include crowding of the bronchi, loss of definition of the bronchovascular markings, oligaemia and, in more severe disease, honeycombing, air-fluid levels or fluid-filled nodules. So, careful examination of chest radiographs is important. ${ }^{54}$ When CT appeared as a diagnostic tool, it replaced bronchography as the 'gold standard' for the diagnosis of bronchiectasis. Cylindrical malformation appears as 'tram lines' or, when imaged in cross-section, as thick-walled circular structures called 'signet rings'. Varicose bronchiectasis has a typical beaded appearance, and saccular or cystic changes appear as a string of cysts, a cluster of grapes, or even as air-fluid levels due to retained secretions (figure 1).

For evaluation of the severity of bronchiectasis by $\mathrm{CT}$, the scoring system of Bhalla et $a l^{55}$ is widely used. Bronchiectasis is present when the internal luminal diameter is slightly greater than the adjacent blood vessel, and peribronchial thickening is present when the wall thickness is equal to or larger than the 
Figure 1 (A) Cylindrical bronchiectasis appearing as a 'signet ring' (arrow) and 'tram lines' (dashed arrow). (B) Varicose bronchiectasis (arrow). (C) Cystic deformation of bronchi (arrow) combined with various bronchiectasis with beaded appearance (dashed arrow).
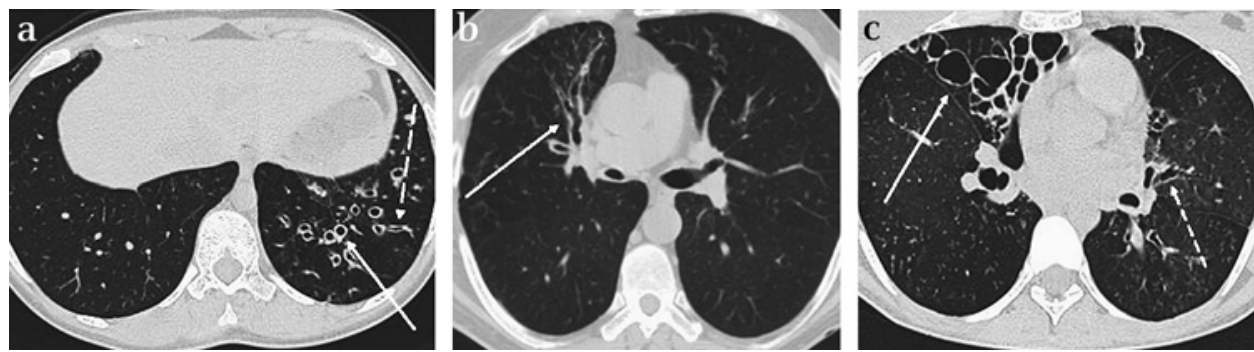

diameter of the adjacent vessel. Further scoring is performed by evaluating the extent of mucus plugging, the extent of bronchiectasis, the presence of abscesses or sacculation, the generation of bronchial divisions involved, the number of bullae, the presence of emphysema, collapse, and/or consolidation.

If patients are evaluated with HRCT, one has to be aware that exacerbation status is important. Shah et a ${ }^{56}$ found that air-fluid levels, mucus plugging, centrilobular nodules and peribronchial thickening were potentially reversible findings in symptomatic patients with bronchiectasis. Even more striking is the fact that bronchiectasis is often reported in children in cases where localised but reversible bronchial dilatation is present, and they are often incorrectly labelled as having an irreversible lifelong condition. Therefore Gaillard et $a l^{57}$ suggest that, in children, a diagnosis of established bronchiectasis should only be made in the presence of a combination of persistent clinical signs and persistent change on an interval CT scan. This interval scan could be performed up to 2 years after the first, at a time when the child is well and free from acute infection.

In cases where radiation is relatively contraindicated (eg, children, pregnancy), a recent study showed that chest high-field 3.0 T MRI appears to be as effective as HRCT in assessing the extent and severity of lung abnormalities in non-CF lung disease $^{58}$

\section{Diagnostic work-up}

Diagnosing bronchiectasis on radiological imaging needs further diagnostic work-up to reveal the underlying cause. CF should be considered when young patients ( $<40$ years) with clinical features of malabsorption, a history of infertility, upper lobe bronchiectasis or sputum organisms typically identified in CF (Staph aureus and/or Ps aeruginosa) are encountered. Exclusion of $\mathrm{CF}$ requires a negative sweat test and the absence of two CFTR mutations, and, in some cases, additional testing by means of nasal potential difference measurement may be indicated.

When dealing with NCFB, other tests may indicate the underlying aetiology (table 2), including a detailed patient history, biochemical evaluation of venous blood sample, radiology, pulmonary function tests, sputum investigations and ciliary function tests. ${ }^{42} 59$

\section{CURRENT CONTROVERSIES IN THE MANAGEMENT OF NCFB}

The goal of treatment is to improve the symptoms of cough, sputum production, and dyspnoea, and to prevent the progression of airway damage. Consensus on several therapeutic strategies that have been evaluated in CF and COPD is lacking in NCFB, and a number of controversies need further elucidation.

\section{Vaccination}

In many countries, influenza vaccination is part of routine immunisation recommendation in people aged 65 and over and in those with chronic illnesses. There are no randomised controlled trials examining the effectiveness of influenza vaccination in patients with bronchiectasis. ${ }^{60}$ There is limited evidence to support the use of 23 -valent pneumococcal vaccination as routine management in adults and children with bronchiectasis. $^{61}$ As both groups in this trial also received influenza vaccine, we suggest that influenza vaccination should also be administered. With the lack of evidence on how often the vaccine should be given, it is recommended that health providers adhere to national guidelines. ${ }^{62}$

\section{Mobilisation of airway secretions}

The increased production of mucus, together with impairment of the mucociliary system, leads to mucus accumulation, cough and recurrent infections. Patients with NCFB may benefit from interventions aimed to help clear excessive secretions. In contrast with CF, only a small number of studies evaluating this type of treatment have been performed in patients with NCFB. Physiotherapy is regarded as standard treatment when dealing with bronchiectasis, but evidence in NCFB is limited. ${ }^{63} 64$ This comprises postural drainage and techniques such as the active cycle breathing technique.

Several mechanical devices (eg, high frequency chest wall oscillation, oscillatory positive expiratory pressure devices (flutter device, acapella device, positive expiratory pressure mask)) are regarded as standard treatment for patients with CF bronchiectasis, and a limited number of studies have evaluated their use in patients with NCFB. ${ }^{6566}$ A recent randomised crossover study by Murray et al ${ }^{67}$ evaluated 3 months of twice daily chest physiotherapy using an oscillatory positive expiratory pressure device compared with 3 months of no chest physiotherapy in patients with NCFB. There was a significant improvement in the LCQ, $24 \mathrm{~h}$ sputum volume production, exercise capacity and SGRQ total score. No significant differences were seen in sputum bacteriology or pulmonary function parameters. Although there does not seem to be a consensus about their use in NCFB, regular chest physiotherapy may have small, but significant benefits in NCFB

There have been no randomised studies that have validated the usefulness of bronchodilator therapy such as short-acting or long-acting $\beta$-agonists, anticholinergics, theophylline and leukotriene antagonists in the treatment of bronchiectatic patients. ${ }^{68-72}$ However, because many patients with bronchiectasis also have COPD or show airflow obstruction and bronchial hyper-reactivity, patients commonly receive these treatments.

Inhaled mucolytics such as erdosteine (a derivative of the mucoactive compound thiol) showed, in combination with physiotherapy, $\mathrm{FEV}_{1}$ improvement of an average $200 \mathrm{ml}^{73}$ Another inhaled mucolytic, recombinant human DNase (rhDNase), had a negative effect on $\mathrm{FEV}_{1}$ and should thus probably not be used in clinical practice for NCFB. The explanation for the opposite effect of rhDNase in NCFB versus CF 
Table 2 Further investigations, suggestive signs and expected abnormal findings according to the underlying aetiology

\begin{tabular}{ll}
\hline Aetiology & Suggestive signs \\
\hline Cystic fibrosis & $\begin{array}{l}\text { Age under } 40, \text { malabsorption, poor } \\
\text { growth, infertility in males, faecal masses } \\
\text { on abdominal x-ray, diabetes }\end{array}$ \\
Congenital disorders & $\begin{array}{l}\text { Primary ciliary dyskinesia: sinusitis, otitis } \\
\text { media, hearing loss, poor sense of smell, } \\
\text { middle lobe predominance }\end{array}$
\end{tabular}

Additional inve
Sweat test
Genetic testing
NPD
Nasal epithelial

Nasal epithelial brushing or biopsy

Nasal NO measurement ( $>5$ years of age) Saccharin test (no clinical value anymore)

Marfan's syndrome: myopia, arachnodactylia, tall stature, thoracic deformations, glaucoma, abnormal joint flexibility, heart murmur

\section{a1-Antitrypsin deficiency}

Anatomical deformations: visible on clinical examination

IBD

Coeliac disease

Post infectious

Immunological disorders

ABPA

Rheumatic disorders (RA, SLE, Sjögren ankylosing spondylitis, relapsing polychondritis)

\section{COPD}

Traction, obstruction, inhalation

YNS, Young's syndrome, amyloidosis, endometriosis

Idiopathic
Diarrhoea, abdominal pain, haematochezia, weight loss, arthritis, pyoderma gangrenosum, primary sclerosing cholangitis

Malabsorption, chronic diarrhoea, failure to thrive in children, fatigue, mouth ulcers, anaemia, weight loss, dermatitis herpetiformis

History of multiple pulmonary infections, tuberculosis or cough suppression

Primary: recurrent infections, developmental delay in children, particular organ problems

Secondary: lung transplant patients, patients under immunosuppressive therapy, HIV

Asthma, wheezing, coughing up brownish mucoid plugs or blood, upper lobe predominance

RA: rheumatoid nodule, arthritis, synovitis, specific skeletal deformities, rheumatoid nodule, other skin symptoms, etc

SLE: malar rash, ulcers, neuropsychiatric symptoms, etc

Dyspnoea, Smoking history, Recurrent infections

Sarcoïdosis: fatigue, erythema nodosum, lupus pernio, arthralgia, uveitis, Bell's palsy, etc

History of radiation therapy

History of inhalation/aspiration trauma

YNS: yellow dystrophic nails, lymphoedema, sinusitis, pleural effusion Young's syndrome: history of mercury contact, rhinosinusitis, infertility

Endometriosis: pelvic pain, infertility, cyclic haemoptysis/pain

Lower lobe predominance, combined chronic rhinitis/sinusitis
Search for major and minor indicators of the disorder
a1-Antitrypsin deficiency
Thoracic imaging

Colonoscopy with biopsy of pathological lesions

tTG antibodies and $\operatorname{Ig} A$

Endoscopic duodenal or jenunal biopsies

History or radiological evidence of previous infection

Sputum with smear and culture for acidfast bacilli

$\lg \mathrm{G}$ and subclasses, $\lg \mathrm{A}, \lg \mathrm{M}$

Full blood count

Neutrophil antibody and function test, challenge with common humoral bacterial antigens

$\lg \mathrm{G}$ and subclasses, $\lg \mathrm{A}, \lg \mathrm{M}$

HIV testing

Total IgE, sputum sample

Specific serum IgE and IgG to Aspergillus fumigatus

Aspergillus fumigatus skin prick test

Autoimmune screening: rheumatoid

factor, ANCAs, ANAs and anti-

citrullinated peptide antibodies

Rheumatological advice

Spirometry, bronchodilatation test

Chest CT scan

Biopsy

Bronchoscopy if imaging showing foreign body

Exclusion diagnosis based on imaging and clinical findings

Urological advice

Gynaecological evaluation

All of the above excluded
Gastrointestinal advice

Expected abnormalities

Positive sweat test: chloride concentration $>60 \mathrm{mEq} / \mathrm{l}$

2 CFTR mutations

Abnormal NPD

Abnormal ciliary beat pattern and frequency of ciliogenesis in culture

Nasal NO $<150 \mathrm{ppb}$

Increased time ( $>60 \mathrm{~min}$ ) before tasting saccharin

ABPA, allergic brochopulmonary aspergillosis; ANA, anti-nuclear antibodies; ANCA, anti-neutrophil cytoplasmic antibodies; CFTR, cystic fibrosis transmembrane conductance regulator; COPD, chronic obstructive pulmonary disease; IBD, inflammatory bowel disease; NPD, nasal potential difference; RA, rheumatoid arthritis; SLE, systemic lupus erythematosus; tTG antibodies, tissue transglutaminase antibodies; YNS, yellow nail syndrome. 
remains unclear. Inhaled hyperosmolar agents such as dry powder mannitol and hypertonic saline have also been proposed. Preliminary results for nebulised hypertonic saline solution (7\%) have shown promise in the treatment of patients with both $\mathrm{CF}$ and NCFB, but long-term prospective trials are needed. ${ }^{74} 75$ An uncontrolled short-term study showed that inhaled mannitol over 12 days reduced the tenacity, acutely increased the hydration of mucus, and improved cough clearability in patients with NCFB. Despite the lack of additional evidence, these agents are increasingly used in routine clinical practice. ${ }^{75-77}$

\section{Antimicrobial therapy}

Antimicrobial therapy, mostly antibiotics, has been tried systemically and via inhalation. When systemic antibiotics are used, strategies applied differ greatly. Sometimes they are used as needed or schematically, alternating one or more antibiotics. Overall, chronic antibiotic treatment is currently not recommended. There is one exception: macrolide therapy. Owing to their non-antibiotic effect on bronchiectatic inflammation, the use of macrolides is very interesting. Total BAL cell count, sputum volume, interleukin (IL)-8 and neutrophils all seem to decrease with macrolide use. Patients with frequent exacerbations of NCFB who had been treated with rescue antibiotics showed an improvement in exacerbation frequency, spirometry and sputum microbiology when treated with azithromycin $250 \mathrm{mg}$ three times a week. ${ }^{78}$ This explains the current widespread use of macrolide therapy for bronchiectatic patients. Despite the fact that inhaled antibiotics have more limited side effects, they are more expensive and may be less well tolerated because of bronchospasm. Nebulised tobramycin decreased the number of admissions and days in hospital. A Cochrane review showed a possible positive effect of prolonged (more than 4 weeks) antibiotic use for bronchiectasis in selected patients. ${ }^{79}$ Overall, antibiotics are rarely used in clinical practice for NCFB, but in patients with CF bronchiectasis, treatment with aerosolised anti-pseudomonal antibiotics is recommended. $^{80}$

Furthermore, assessing sputum colour and taking sputum culture should be performed regularly. It is generally accepted that earlier diagnosis, close monitoring and intensive therapy reduces the frequency and severity of exacerbations and seems to slow pulmonary decline. This is probably explained by the delay in colonisation due to effective treatment of acute infections.

\section{Anti-Inflammatory agents}

Generally, with the exception of macrolides, anti-inflammatory strategies lack evidence and need bigger trials with the right end points. For example, oral corticoid therapy in NCFB has never been studied in a controlled manner, although there are some studies suggesting that inhaled steroids reduce sputum volume and improve health-related quality of life questionnaire results but have no effect on $\mathrm{FEV}_{1}$ and exacerbation frequency. ${ }^{81-83}$ Indomethacin, on the other hand, showed a reduction in peripheral neutrophil chemotaxis even though there was no change in exacerbation, elastase, myeloperoxidase or sputum albumin. Overall, there is a lack evidence for the efficacy of systemic corticoids and ibuprofen in NCFB and CF bronchiectasis because of important side effects.

A large range of experimental products have been tried in animal models, such as aerolised IL-1 receptor antagonist and F $(\mathrm{ab})_{2}$ from IL-8 monoclonal antibody, but are currently not indicated in routine clinical practice. Further research on antiinflammatory agents is necessary.

\section{Surgical treatment}

Surgical intervention is rarely needed and appears to have an acceptable morbidity and mortality in selected patients with NCFB. The indications for surgical resection are the presence of localised areas of cystic, non-perfused bronchiectasis with symptoms that do not respond to conservative treatment, and life-threatening complications such as severe uncontrolled haemoptysis, despite the use of bronchial artery embolisation, or pulmonary abscesses. Pseudomonas infection and underlying chronic obstructive airway disease are poor prognostic factors for surgical intervention.

End-stage bronchiectasis has been successfully treated with lung transplantation, preferably with double-lung transplants.

\section{Patients treated with biological response modifiers (BRMs)}

When BRMs emerged, adverse effects accompanied their use, in particular infectious complications. In this respect, the question arises whether the presence of bronchiectasis should be a contraindication to the use of BRMs. Lieberman-Maran et al ${ }^{84}$ address this problem, reviewing present literature and adding their own experience. For etanercept, no analysis was included in the literature that directly addressed infections in patients with underlying lung disease, including bronchiectasis. For infliximab, the presence of bronchiectasis was an exclusion criterion. Conversely, bronchiectasis was not specifically mentioned as an exclusion criterion for anakinra trials. When the safety data of anakinra were analysed, infections were not classified relative to the presence or absence of bronchiectasis. Finally, adalimumab did not exclude patients on the basis of the presence of bronchiectasis. The Abbott Laboratory database contained no information on adalimumab regarding serious adverse events related to the presence of bronchiectasis. The authors conclude that patients with rheumatoid arthritis who are being considered for treatment with BRMs should be evaluated for the presence of bronchiectasis before such therapy is initiated if there is a clinical suspicion of underlying pulmonary disease. Furthermore, caution should be exercised in initiating these types of therapy in patients with bronchiectasis. We suggest that, in patients with recurrent episodes of pneumonia and bronchitis, BRMs be avoided until more trials have been carried out. We not only recommend this for rheumatoid arthritis, but also for other patients for whom BRMs are considered.

\section{Main messages}

- For the diagnosis of bronchiectasis, high-resolution chest (HRCT) is necessary, although MRI is a good alternative in cases where radiation must be avoided.

- HRCT findings during a respiratory exacerbation can be different from findings during stable situation.

- Diagnosing bronchiectasis in children should be performed with great caution, as the bronchodilatation can be reversible. Imaging should be repeated later on in the absence of an acute infection.

- Patients should have a diagnostic work-up, searching for underlying disease.

- The Leicester Cough Questionnaire, sputum colour chart and sputum screening should be performed on a regular basis.

- Treatment of non-cystic fibrosis bronchiectasis should consist of smoking cessation, vaccination, physiotherapy, bronchodilators if necessary and macrolide therapy if needed. Inhaled hyperosmolar agents may also be useful. Acute exacerbations need to be treated with antibiotics. 


\section{Current research questions}

Need for more prevalence and incidence studies evaluating different aetiologies and estimating economic impact on healthcare.

- Are the underlying pathophysiological mechanisms driving lung inflammation the same for the various aetiologies of NCFB? Are they different in CF?

- Need for new biomarkers which will allow a faster diagnosis of infection, resulting in rapid treatment.

- Research for medical therapies with more randomised controlled trials for the treatment of NCFB.

- Should the presence of bronchiectasis be a contraindication to the use of biological response modifiers? Future clinical trials with biological response modifiers should attempt to better stratify risk for individual patients.

\section{Acute exacerbation}

There is no real consensus on how to treat an exacerbation, but immediate antibiotic treatment is necessary. The choice of antibiotics should depend on the organism found, and $10-14$ days seems to be an acceptable duration of treatment. ${ }^{85}$ The agent chosen should be active against Hae influenzae and Staph aureus. Non-pseudomonal antibiotics could be used for patients who usually produce little sputum and have good lung function. For patients with more severe disease, recent antibiotic treatment or CF, an anti-pseudomonal treatment should also be considered. $^{86}$ Anti-pseudomonal antibiotics include aminoglycosides, ceftazidime or carbapenems, and newer quinolones, although there is increasing resistance to the latter. Non-pseudomonal options are third-generation cephalosporins and $\beta$ lactams with lactamase inhibitor such as amoxicillin with clavulanic acid or macrolides. ${ }^{21}$ Sputum culture should be performed with sensitivity testing. The results of this testing and patient response to treatment should guide further management. Furthermore, bronchodilator therapy can be added in the case of obstruction or symptoms as can steroid therapy, although there are no randomised controlled trials on this. Finally, high doses of bromhexine with antibiotics has been found to ease difficulty in expectoration sputum. ${ }^{87}$

Despite some improvement in the evidence-based guide to management in NCFB, there remains a serious lack of randomised controlled trials directing therapy.

\section{Key references}

- Murray MP, Turnbull K, MacQuarrie S, et al. Validation of the Leicester Cough Questionnaire in non-cystic fibrosis bronchiectasis. Eur Respir J 2009;34:125-31.

- Fuschillo S, De FA, Balzano G. Mucosal inflammation in idiopathic bronchiectasis: cellular and molecular mechanisms. Eur Respir J 2008;31:396-406.

- Murray MP, Hill AT. Non-cystic fibrosis bronchiectasis. Clin Med 2009;9:164-9.

- Tsang KW, Bilton D. Clinical challenges in managing bronchiectasis. Respirology 2009;14:637-50.

- Anwar GA, Bourke SC, Afolabi G, et al. Effects of long-term low-dose azithromycin in patients with non-CF bronchiectasis. Respir Med 2008;102:1494-6.

\section{SUMMARY}

On finding bronchiectasis, it is necessary to look for the underlying aetiology, usually excluding CF. Afterwards, patients need individualised treatment and follow-up by monitoring of their symptoms, for example, with the LCO and sputum colour chart. Screening patients for bacterial colonisation on a regular basis is important, as many become colonised with pathogenic microorganisms. Treatment for NCFB differs in certain aspects from that for CF bronchiectasis and often lacks evidence. Overall, bronchiectasis is an underestimated disease, not only in prevalence and incidence, but also in its ability to cause great morbidity and mortality. Further research into the underlying pathophysiological mechanisms in NCFB, and whether these differ from one aetiology to another, is necessary.

\section{MULTIPLE-CHOICE QUESTIONS (TRUE (T)/FALSE (F); ANSWERS AFTER THE REFERENCES)}

1. Concerning the symptoms of bronchiectasis:

A. Factors associated with disease progression are a history of severe exacerbations, chronic colonisation with Pseudomonas aeruginosa and evidence of systemic inflammation.

B. Severe NCFB may also induce pulmonary hypertension and right and left ventricular systolic and diastolic dysfunction.

C. Loss of lung function has been demonstrated in patients with non-smoking NCFB, with an average decline of $\sim 50 \mathrm{ml}$ /year in $\mathrm{FEV}_{1}$.

D. The high prevalence in some areas is probably due to a racial or geographical predisposition.

\section{Concerning the follow-up of bronchiectasis:}

A. The LCQ is a validated tool for assessing cough impact in a standardised way. It is able to discriminate disease severity and to document treatment response.

B. For the microbiological analysis, BAL is superior to protected specimen brush and both BAL and protected specimen brush are superior to spontaneous or induced sputum analysis.

C. The SGRO is another validated but longer health-related questionnaire that can be used in the follow-up of bronchiectasis.

D. In the follow-up of bronchiectasis, the sputum colour chart is useful for assessing bacterial colonisation.

\section{Concerning the imaging of bronchiectasis:}

A. For children, the diagnosis of established bronchiectasis should only be made according to a combination of persistent clinical signs and persistent change on an interval CT scan.

B. Air-fluid levels, mucus plugging, centrilobular nodules and peribronchial thickening are potentially reversible findings in symptomatic patients with bronchiectasis.

C. Careful examination of chest radiographs is important, as one study suggested that chest radiography was normal in only $7.1 \%$ of patients.

D. MRI appears to be less effective than HRCT in assessing the extent and severity of lung abnormalities in non-CF lung disease.

\section{Concerning therapeutic options:}

A. The use of 23-valent pneumococcal vaccination as routine management together with an influenza vaccination is recommended in patients with bronchiectasis.

B. There is no evidence that patients may benefit from regular chest physiotherapy. 
C. Patients with frequent exacerbation of NCFB who were treated with rescue antibiotics showed an improvement in exacerbation frequency, spirometry and sputum microbiology when treated with azithromycin $250 \mathrm{mg}$ three times a week.

D. Patients with localised areas of cystic, non-perfused bronchiectasis with symptoms that do not respond to conservative treatment, may benefit from surgical intervention.

\section{Concerning controversies and therapy in bronchiectasis:}

A. Although recombinant human DNase showed good effect in CF bronchiectasis, it is not recommended for NCFB.

B. Patients who are being considered for therapy with biological response modifiers should be evaluated for the presence of bronchiectasis before initiating such therapy if there is clinical suspicion of underlying pulmonary disease.

C. Although there is no real evidence, bronchodilator therapy such as short-acting or long-acting $\beta$-agonists, anticholinergics, theophylline and leukotriene antagonists can be suggested in selected bronchiectatic patients.

D. Systemic corticoid therapy is an established treatment option for NCFB. Ibuprofen should be avoided because of important side effects.

\section{Competing interests None}

Provenance and peer review Not commissioned; externally peer reviewed.

\section{REFERENCES}

1. Bilton D. Update on non-cystic fibrosis bronchiectasis. Curr Opin Pulm Med 2008;14:595-99.

2. Roguin A. Rene Theophile Hyacinthe Laënnec (1781-1826): the man behind the stethoscope. Clin Med Res 2006;4:230-35.

3. Wrong 0. Osler and my father. J R Soc Med 2003;96:462-64.

4. Fall A, Spencer D. Paediatric bronchiectasis in Europe: what now and where next? Paediatr Respir Rev 2006;7:268-74.

5. Singleton R, Morris A, Redding G, et al. Bronchiectasis in Alaska Native children: causes and clinical courses. Pediatr Pulmonol 2000;29:182-87.

6. Twiss $\mathbf{J}$, Metcalfe R, Edwards E, et al. New Zealand national incidence of bronchiectasis "too high" for a developed country. Arch Dis Child 2005:90:737-40.

7. O'Donnell AE, Barker AF, llowite JS, et al. Treatment of idiopathic bronchiectasis with aerosolized recombinant human DNase I. rhDNase Study Group. Chest 1998:113:1329-34.

8. Martínez-García MA, Soler-Cataluna JJ, Perpiñà-Tordera M, et al. Factors associated with lung function decline in adult patients with stable non-cystic fibrosis bronchiectasis. Chest 2007;132:1565-72.

9. King PT, Holdsworth SR, Freezer NJ, et al. Outcome in adult bronchiectasis. COPD 2005:2:27-34

10. Alzeer AH, Al-Mobeirek AF, Al-Otair HA, et al. Right and left ventricular function and pulmonary artery pressure in patients with bronchiectasis. Chest 2008;133:468-73.

11. Wilson CB, Jones PW, O'Leary CJ, et al. Validation of the St. George's Respiratory Questionnaire in bronchiectasis. Am J Respir Crit Care Med 1997;156:536-41.

12. Murray MP, Turnbull K, MacQuarrie S, et al. Validation of the Leicester Cough Questionnaire in non-cystic fibrosis bronchiectasis. Eur Respir J 2009;34:125-31.

13. Fuschillo $\mathbf{S}$, De FA, Balzano G. Mucosal inflammation in idiopathic bronchiectasis: cellular and molecular mechanisms. Eur Respir J 2008;31:396-406.

14. Paulson ML, Freeman AF, Holland SM. Hyper IgE syndrome: an update on clinical aspects and the role of signal transducer and activator of transcription 3. Curr Opin Allergy Clin Immunol 2008:8:527-33.

15. Dhillon SS, Watanakunakorn C. Lady Windermere syndrome: middle lobe bronchiectasis and Mycobacterium avium complex infection due to voluntary cough suppression. Clin Infect Dis 2000;30:572-75.

16. King PT. The pathophysiology of bronchiectasis. Int J Chron Obstruct Pulmon Dis 2009:4:411-19.

17. O'Brien C, Guest PJ, Hill SL, et al. Physiological and radiological characterisation of patients diagnosed with chronic obstructive pulmonary disease in primary care. Thorax 2000;55:635-42.

18. Patel IS, Vlahos I, Wilkinson TM, et al. Bronchiectasis, exacerbation indices, and inflammation in chronic obstructive pulmonary disease. Am J Respir Crit Care Med 2004:170:400-7.

19. Kim RD, Greenberg DE, Ehrmantraut ME, et al. Pulmonary nontuberculous mycobacterial disease: prospective study of a distinct preexisting syndrome. Am J Respir Crit Care Med 2008:178:1066-74.

20. Fajac I, Viel M, Sublemontier $S$, et al. Could a defective epithelial sodium channel lead to bronchiectasis. Respir Res 2008:9:46.
21. Tsang KW, Bilton D. Clinical challenges in managing bronchiectasis. Respirology 2009;14:637-50.

22. Lee $\mathbf{A B}$, Bogaars HA, Passero MA. Nodular pulmonary amyloidosis. A cause of bronchiectasis and fatal pulmonary hemorrhage. Arch Intern Med 1983:143:603-4.

23. McMahon MJ, Swinson DR, Shettar S, et al. Bronchiectasis and rheumatoid arthritis: a clinical study. Ann Rheum Dis 1993:52:776-79.

24. Shadick NA, Fanta CH, Weinblatt ME, et al. Bronchiectasis.A late feature of severe rheumatoid arthritis. Medicine (Baltimore) 1994;73:161-70.

25. Hassan WU, Keaney NP. Holland CD, et al. High resolution computed tomography of the lung in lifelong non-smoking patients with rheumatoid arthritis. Ann Rheum Dis 1995; 54:308-10.

26. Cortet B, Flipo RM, Rémy-Jardin M, et al. Use of high resolution computed tomography of the lungs in patients with rheumatoid arthritis. Ann Rheum Dis 1995:54:815-19.

27. Clause HP, Sanger PW, Taylor FH, et al. Systemic lupus erythematosus associated with bronchiectasis: report of two cases. Dis Chest 1964;45:219-22.

28. Mahadeva R, Flower C, Shneerson J. Bronchiectasis in association with coeliac disease. Thorax 1998;53:527-29.

29. Shi JH, Liu HR, Xu WB, et al. Pulmonary manifestations of Sjogren's syndrome. Respiration 2009:78:377-86.

30. Butler H, Lake KB, Van Dyke JJ. Bronchial endometriosis and bronchiectasis. A possible relationship. Arch Intern Med 1978;138:991-92.

31. Camus $\mathbf{P}$, Colby TV. The lung in inflammatory bowel disease. Eur Respir $J$ 2000;15:5-10

32. Black H, Mendoza M, Murin S. Thoracic manifestations of inflammatory bowel disease. Chest 2007:131:524-32.

33. Cohen M, Sahn SA. Bronchiectasis in systemic diseases. Chest 1999:116:1063-74.

34. Raj AA, Birring SS, Green R, et al. Prevalence of inflammatory bowel disease in patients with airways disease. Respir Med 2008;102:780-85

35. Guilemany JM, Mullol J, Picado C. [Relation between rhinosinusitis and bronchiectasis]. Arch Bronconeumol 2006;42:135-40.

36. Arya AK, Beer HL, Benton J, et al. Does Young's syndrome exist? J Laryngol Otol 2009:123:477-81.

37. Gülhan $\mathbf{M}$, Ozyilmaz E, Tarhan $\mathrm{G}$, et al. Helicobacter pylori in bronchiectasis: a polymerase chain reaction assay in bronchoalveolar lavage fluid and bronchiectatic lung tissue. Arch Med Res 2007;38:317-21.

38. Kanbay M, Kanbay A, Boyacioglu S. Helicobacter pylori infection as a possible risk factor for respiratory system disease: a review of the literature. Respir Med 2007; 101:203-09.

39. Angrill J, Sánchez N, Agusti C, et al. Does Helicobacter pylori have a pathogenic role in bronchiectasis? Respir Med 2006;100:1202-7.

40. Tsang KW, Lam WK, Kwok E, et al. Helicobacter pylori and upper gastrointestinal symptoms in bronchiectasis. Eur Respir J 1999;14:1345-50.

41. Yalçin E, Ozçelik U, Engin D, et al. Does Helicobacter pylori cause bronchiectasis? Acta Paediatr 2002;91:1403.

42. Pasteur MC, Helliwell SM, Houghton SJ, et al. An investigation into causative factors in patients with bronchiectasis. Am J Respir Crit Care Med 2000;162:1277-84

43. Li AM, Sonnappa S, Lex C, et al. Non-CF bronchiectasis: does knowing the aetiology lead to changes in management? Eur Respir J 2005;26:8-14.

44. Eastham KM, Fall AJ, Mitchell L, et al. The need to redefine non-cystic fibrosis bronchiectasis in childhood. Thorax 2004;59:324-24.

45. Angrill J, Agusti C, de CR, et al. Bacterial colonisation in patients with bronchiectasis: microbiological pattern and risk factors. Thorax 2002:57:15-19.

46. King PT, Holdsworth SR, Freezer NJ, et al. Microbiologic follow-up study in adult bronchiectasis. Respir Med 2007;101:1633-38.

47. Angrill J, Agusti C, de CR, et al. Bronchial inflammation and colonization in patients with clinically stable bronchiectasis. Am J Respir Crit Care Med 2001;164:1628-32.

48. Fowler SJ, French J, Screaton NJ, et al. Nontuberculous mycobacteria in bronchiectasis: Prevalence and patient characteristics. Eur Respir J 2006;28:1204-10.

49. Wickremasinghe $\mathbf{M}$, Ozerovitch LJ, Davies G, et al. Non-tuberculous mycobacteria in patients with bronchiectasis. Thorax 2005;60:1045-51.

50. Kunst H, Wickremasinghe M, Wells A, et al. Nontuberculous mycobacterial disease and Aspergillus-related lung disease in bronchiectasis. Eur Respir $J$ 2006:28:352-57.

51. Ledson MJ, Gallagher MJ, Walshaw MJ. Chronic Burkholderia cepacia bronchiectasis in a non-cystic fibrosis individual. Thorax 1998:53:430-32.

52. Murray MP, Pentland JL, Turnbull K, et al. Sputum colour: a useful clinical tool in non-cystic fibrosis bronchiectasis. Eur Respir J 2009;34:361-64.

53. Reid LM. Reduction in bronchial subdivision in bronchiectasis. Thorax 1950;5:233-47.

54. Aronchick JM, Miller WT Jr. Bronchiectasis. J Thorac Imaging 1995;10:255-67

55. Bhalla M, Turcios N, Aponte V, et al. Cystic fibrosis: scoring system with thinsection CT. Radiology 1991;179:783-88.

56. Shah RM, Sexauer W, Ostrum BJ, et al. High-resolution CT in the acute exacerbation of cystic fibrosis: evaluation of acute findings, reversibility of those findings, and clinical correlation. AJR Am J Roentgenol 1997;169:375-80.

57. Gaillard EA, Carty $\mathrm{H}$, Heaf D, et al. Reversible bronchial dilatation in children: comparison of serial high-resolution computer tomography scans of the lungs. Eur $J$ Radiol 2003;47:215-20. 
58. Montella S, Santamaria F, Salvatore M, et al. Assessment of chest high-field magnetic resonance imaging in children and young adults with noncystic fibrosis chronic lung disease: comparison to high-resolution computed tomography and correlation with pulmonary function. Invest Radiol 2009:44:532-38.

59. Murray MP, Hill AT. Non-cystic fibrosis bronchiectasis. Clin Med 2009;9:164-69.

60. Chang CC, Morris PS, Chang AB. Influenza vaccine for children and adults with bronchiectasis. Cochrane Database Syst Rev 2007;(3):CD006218.

61. Furumoto A, Ohkusa $Y$, Chen $M$, et al. Additive effect of pneumococcal vaccine and influenza vaccine on acute exacerbation in patients with chronic lung disease. Vaccine 2008;26:4284-89.

62. Chang CC, Singleton RJ, Morris PS, et al. Pneumococcal vaccines for children and adults with bronchiectasis. Cochrane Database Syst Rev 2009;(2):CD006316.

63. Patterson JE, Bradley JM, Elborn JS. Airway clearance in bronchiectasis: a randomized crossover trial of active cycle of breathing techniques (incorporating postural drainage and vibration) versus test of incremental respiratory endurance. Chron Respir Dis 2004;1:127-30.

64. Mutalithas K, Watkin G, Willig B, et al. Improvement in health status following bronchopulmonary hygiene physical therapy in patients with bronchiectasis. Respir Med 2008;102:1140-44.

65. Patterson JE, Hewitt 0 , Kent L, et al. Acapella versus 'usual airway clearance during acute exacerbation in bronchiectasis: a randomized crossover trial. Chron Respir Dis 2007:4:67-74.

66. Eaton T, Young P, Zeng I, et al. A randomized evaluation of the acute efficacy, acceptability and tolerability of flutter and active cycle of breathing with and without postural drainage in non-cystic fibrosis bronchiectasis. Chron Respir Dis 2007:4:23-30.

67. Murray MP, Pentland JL, Hill AT. A randomised crossover trial of chest physiotherapy in non-cystic fibrosis bronchiectasis. Eur Respir J 2009:34:1086-92.

68. Franco F, Sheikh A, Greenstone M. Short acting beta-2 agonists for bronchiectasis. Cochrane Database Syst Rev 2003;(3):CD003572.

69. Sheikh A, Nolan D, Greenstone M. Long-acting beta-2-agonists for bronchiectasis. Cochrane Database Syst Rev 2001;(4):CD002155.

70. Corless JA, Warburton CJ. Leukotriene receptor antagonists for non-cystic fibrosis bronchiectasis. Cochrane Database Syst Rev 2000;(4):CD002174.

71. Steele K, Greenstone M, Lasserson JA. Oral methyl-xanthines for bronchiectasis. Cochrane Database Syst Rev 2001;(1):CD002734.

72. Lasserson T, Holt K, Evans D, et al. Anticholinergic therapy for bronchiectasis. Cochrane Database Syst Rev 2001;(4):CD002163.

73. Crisafulli E, Coletti 0 , Costi $\mathrm{S}$, et al. Effectiveness of erdosteine in elderly patients with bronchiectasis and hypersecretion: a 15-day, prospective, parallel, open-label, pilot study. Clin Ther 2007;29:2001-09.

74. Elkins MR, Bye PT. Inhaled hypertonic saline as a therapy for cystic fibrosis. Cur Opin Pulm Med 2006;12:445-52.
75. Kellett F, Redfern J, Niven RM. Evaluation of nebulised hypertonic saline (7\%) as an adjunct to physiotherapy in patients with stable bronchiectasis. Respir Med 2005;99:27-31.

76. Daviskas $\mathbf{E}$, Anderson SD, Eberl S, et al. Effect of increasing doses of mannitol on mucus clearance in patients with bronchiectasis. Eur Respir J 2008;31:765-72.

77. Wills $\mathbf{P}$, Greenstone M. Inhaled hyperosmolar agents for bronchiectasis. Cochrane Database Syst Rev 2006;(2):CD002996.

78. Anwar GA, Bourke SC, Afolabi G, et al. Effects of long-term low-dose azithromycin in patients with non-CF bronchiectasis. Respir Med 2008;102:1494-96.

79. Evans DJ, Bara Al, Greenstone M. Prolonged antibiotics for purulent bronchiectasis in children and adults. Cochrane Database Syst Rev 2007;(2):CD001392.

80. Rosen MJ. Chronic cough due to bronchiectasis: ACCP evidence-based clinical practice guidelines. Chest 2006;129(1 Suppl):122S-31S.

81. Martinez-Garcia MA, Perpina-Tordera M, Roman-Sanchez P, et al. Inhaled steroids improve quality of life in patients with steady-state bronchiectasis. Respir Med 2006;100:1623-32

82. Tsang KW, Tan KC, Ho PL, et al. Inhaled fluticasone in bronchiectasis: a 12 month study. Thorax 2005;60:239-43.

83. Elborn JS, Johnston B, Allen F, et al. Inhaled steroids in patients with bronchiectasis. Respir Med 1992;86:121-24.

84. Lieberman-Maran L, Orzano IM, Passero MA, et al. Bronchiectasis in rheumatoid arthritis: report of four cases and a review of the literature-implications for management with biologic response modifiers. Semin Arthritis Rheum 2006:35:379-87.

85. Tsang KW, Chan WM, Ho PL, et al. A comparative study on the efficacy of levofloxacin and ceftazidime in acute exacerbation of bronchiectasis. Eur Respir $J$ 1999:14:1206-09.

86. Irwin RS, Boulet LP, Cloutier MM, et al. Managing cough as a defense mechanism and as a symptom. A consensus panel report of the American College of Chest Physicians. Chest 1998;114(2 Suppl Managing):133S-81S.

87. Crockett AJ, Cranston JM, Latimer KM, et al. Mucolytics for bronchiectasis. Cochrane Database Syst Rev 2001:(2):CD001289.

\section{ANSWERS}

1. $A(T) ; B(T) ; C(T) ; D(F)$

2. $A(T) ; B(F) ; C(T) ; D(T)$

3. $A(T) ; B(T) ; C(T) ; D(F)$

4. $A(T) ; B(F) ; C(T) ; D(T)$

5. $A(T) ; B(T) ; C(T) ; D(F)$ 